\title{
Wissen schafft Wert
}

\section{Die Christian Doppler Forschungsgesellschaft}

\author{
Online publiziert am 23. Januar 2014
}

\begin{abstract}
Die Christian Doppler Forschungsgesellschaft fördert die Kooperation von hervorragenden WissenschafterInnen und innovativen Unternehmen. Dazu werden an Universitäten und außeruniversitären Forschungseinrichtungen Christian Doppler Labors eingerichtet, die von den beteiligten Unternehmen und der öffentlichen Hand gemeinsam finanziert werden. Größter öffentlicher Fördergeber ist das österreichische Wirtschaftsministerium BMWFJ. Im Zentrum der Kooperation steht anwendungsorientierte Grundlagenforschung.
\end{abstract}

Die Montanuniversität Leoben zeichnet sich seit vieIen Jahren durch eine hohe Anzahl an Christian Doppler Labors aus: Der thematische Bogen reicht von Maschinenund Instrumentenbau über metallische und nichtmetallische Werkstoffe bis zu Chemie. In all diesenThemenfeldern wird herausragende anwendungsorientierte Grundlagenforschung betrieben. In dieser Kooperation von Wissenschaft und Wirtschaft wird Grundlagenwissen erarbeitet, das beim Unternehmenspartner in die Entwicklung neuer Produkte und Verfahren fließt. Dabei erfolgt ein reger Wissens-, Erfahrungs- und Fragenaustausch zwischen den Partnern.

Christian Doppler Labors werden für sieben Jahre eingerichtet und sind für alle Beteiligten ein Gewinn: WissenschafterInnen bauen eine eigene stabile Forschungsgruppe auf und genießen hohe wissenschaftliche Autonomie. Unternehmen gewinnen Grundlagenwissen und können dadurch ihre Innovationsprozesse beschleunigen und vertiefen. Der wissenschaftliche Nachwuchs wird gefördert, und es entstehen strategische Allianzen zwischen Wissenschaft und Wirtschaft. Auf diese Weise wird der Wissenschafts- und Wirtschaftsstandort Österreich in seiner Gesamtheit gestärkt.

Für die Einrichtung eines Christian Doppler Labors müssen zwei Voraussetzungen erfüllt sein: einerseits der konkrete Bedarf eines Unternehmens an Wissen und Know-how aus der anwendungsorientierten Grundlagenforschung und andererseits die Bereitschaft einer Wissenschafterin oder eines Wissenschafters, sich diesem unternehmerischen Bedarf langfristig zu öffnen.

Sind beide Voraussetzungen gegeben, können die Partner gemeinsam ein auf mehrere Jahre angelegtes Forschungsprogramm entwickeln, das einem hohen wissenschaftlichen Anspruch gerecht werden muss. Anträge zur Einrichtung eines Christian Doppler Labors können von WissenschafterInnen jederzeit im Generalsekretariat der Christian Doppler Forschungsgesellschaft eingereicht werden.

\section{Bruno Hribernik}

1. Vizepräsident der Christian Doppler

Forschungsgesellschaft

\section{Mehr Informationen finden Sie unter www.cdg.ac.at}

The Christian Doppler Research Association (CDG) is considered a pioneer in Austria for successful cooperations between science and the private sector. The cooperation funded by the CDG usually looks like this: a research group elaborates on fundamental knowledge that is incorporated in the development of new products and processes of commercial partners. This generates a brisk exchange of knowledge, experience, and ideas for further research between the partners.

More Information: www.cdg.ac.at 